\title{
FORMACIÓN DEL INVESTIGADOR MÁS ALLÁ DE UN CURRÍCULO
}

\section{Marisela Ch. Fernández}

Universidad Nacional Experimental de las Fuerzas Armadas, dmariselachiquinquira@gmail.com

\section{Dilia M. Monasterio}

Universidad Central de Venezuela, ailidadm@gmail.com

\section{Resumen}

El objetivo del estudio fue interpretar la formación de los investigadores en el contexto normativo del currículo y los escenarios sociales de los docentes y participantes que intervienen en los programas de las universidades experimentales en Venezuela. La metodología estuvo enmarcada en una perspectiva interpretativa basada en la hermenéutica, las técnicas fueron de carácter documental, además se recurrió a los escenarios de los sujetos donde se aplicó una entrevista. Los principales hallazgos mostraron la presencia de dos elementos conceptuales: la autoridad y la experiencia que explican la formación de los investigadores. Los significados revelaron que en las universidades el docente es un generador de conocimientos. Mediante su rol de investigador crea teorías que explican la acción educativa y se desempeńa como orientador, mediador e investigador coadyuvando a la formación de los futuros investigadores. En conclusión, el docente se reconoce como la autoridad en los espacios educativos donde se desarrollan los procesos de formación para la investigación. Por lo tanto, el currículo es invisible para el estudiante, el docente en su praxis, representa el medio capaz de visibilizar o no el sentido que otorga el currículo como texto institucional a la formación de los investigadores.

Palabras clave: currículo, formación del investigador, universidades experimentales.

\section{TRAINING THE INVESTIGATOR BEYOND A CURRICULUM}




\section{Abstract}

The objective of the study was to interpret the training of researchers in the normative context of the curriculum and the social scenarios of the teachers and participants who take part in the programs of the Experimental Universities in Venezuela. The methodology was framed in an interpretive perspective based on hermeneutics, the techniques were documentary in nature, in addition to the scenarios of the subjects where an interview was applied. The main findings showed the presence of two conceptual elements: the authority and the experience that explain the training of researchers. The meanings revealed that in universities the teacher is a generator of knowledge, through his role of researcher creates theories that explain the educational action and serves as a guide, mediator and researcher contributing to the training of future researchers. In conclusion, the teacher is recognized as the authority in educational spaces where the processes of training for research are developed. Therefore, the curriculum is invisible to the student, the teacher in his praxis, represents the means capable of making visible or not the sense that the curriculum gives as an institutional text to the training of researchers.

Keywords: curriculum, researcher training, experimental universities.

\section{Introducción}

La década del Siglo XXI se caracteriza por acelerados cambios $\mathrm{y}$ transformaciones en todas partes del mundo, especialmente, en el campo de la ciencia y la tecnología. La sociedad del conocimiento se enrumba hacia enormes progresos que conllevan a la investigación y avance progresivo del conocimiento científico, inspirado en el saber mediante la educación, formación, investigación y desarrollo, programas informáticos y sistemas de información que permiten la creación de nuevos conocimientos. La universidad, como institución social, está permeada por estos avances que marcan el comportamiento de las sociedades; es decir, cambios y reformas en el sector educativo universitario, que buscan transformar al ser humano a través del proceso de formación. Partiendo del principio que la edu- 
cación universitaria es un factor estratégico para el desarrollo del país, se debe profundizar el compromiso con el pueblo, los valores, la capacidad intelectual en todos los ámbitos, la vinculación de proyectos sociales, así como la participación activa y la interacción con los pueblos del mundo.

De esta manera, la educación universitaria en Venezuela enfrenta el reto de superar brechas que existen en materia de formación de investigadores, la poca productividad de la investigación y la cultura investigativa adversa al desarrollo de la investigación científica. Así como también, la carencia del incremento de la inversión pública en ciencia, tecnología e innovación, la formulación de políticas públicas para estimular una creciente inversión por parte de las empresas, incluyendo la formación calificada, el acceso a la información y el equipamiento necesario para el desarrollo de la ciencia. En este contexto, el currículo universitario juega un papel fundamental en la formación del futuro profesional, este implica una construcción, una propuesta y una praxis que se sustenta en supuestos filosóficos, epistemológicos, sociológicos, psicológicos y pedagógicos que se expresan en los diseños curriculares de cada institución educativa, donde la teoría y la práctica constituyen elementos fundamentales en el campo del currículo, a fin de articular en el proceso enseñanza y aprendizaje, la actividad investigativa, académica y laboral.

En la actualidad, este texto institucional llamado diseño curricular, parece responder más al cumplimiento de las exigencias legales y administrativas que demanda el Estado en materia de educación universitaria para formar los profesionales para el mercado laboral que el desarrollo de investigadores. El currículo implica una construcción, una propuesta y una praxis que se sustenta en supuestos filosóficos, epistemológicos, sociológicos, psicológicos y pedagógicos con el fin de quedar claramente explicitados por la institución educativa, donde la teoría y la práctica constituyan los elementos fundamentales del campo del currículo para estar estrechamente articulados en el proceso enseńanza - aprendizaje, mediante la actividad investigativa, académica y laboral. (González, Hernández \& Sanz 2003). Sin embargo, Imbernón (2003), sostiene que en los planes de estudio se tiende a predominar una estructura con muchas asignaturas, fragmentada y parcelada en múltiples cursos lectivos sobre una gran diversidad de materias propiamente 
pedagógicas, profesionales, culturales y con contenidos de otros saberes. Además, los planes se presentan rígidos, tanto en cuanto, a la variedad de la oferta de distintos tipos de actividades curriculares que se expresan por la ausencia de seminarios, talleres, actividades de campo, entre otros elementos. No solo se plantea como una deficiencia en la formación docente, sino que se presenta como una actividad secundaria entre los formadores de formadores. Esto, a pesar de que trabajos recientes valoran la investigación como herramienta importante en la formación docente.

Así mismo, García (2005), señala que la educación se ha centrado en una visión única que es reconocida como la forma verdadera de la investigación, una enseńanza libresca, memorística, verbalista, transmisora de datos fragmentarios e informaciones puntuales que lleva a una comprensión de la ciencia descontextualizada del mundo cotidiano y de las necesidades reales. Por consiguiente, nos enfrentamos a una educación descontextualizada en la formación de los investigadores, partiendo que el currículo universitario deviene de las políticas del Estado venezolano que rige la formación de los educandos en las universidades. En esta línea argumentativa, no cabe duda que las universidades venezolanas poseen una tradición científica desde el año de 1936, cuando se inicia un proceso de creación de centros e instituciones de investigación especializados, particularmente en la Universidad Central de Venezuela. A pesar de esta tradición, no se han logrado los resultados esperados, que contribuyan a desarrollar el país y convertirlo en uno de los primeros lugares en investigación científica. Esto puede ser consecuencia de lo expuesto por Mayz \& Pérez (2002), en relación a que la mayoría de los docentes universitarios no investigan.

Las evidencias señalan que los países que tienen la mayor producción científica en cuanto al número total de publicaciones científicas, indexadas en el Science Citation Index (SCI), son de Argentina, Brasil, Chile y México, factor que refleja una alta productividad en los referidos países, situación contraria en la cual se encuentra Venezuela, su posición en el ranking la ubica por debajo del resto de los países en materia de producción intelectual. En este contexto se evidencia que, a lo largo de este período, la curva de crecimiento del número de publicaciones venezolanas es mucho más plana que la reflejada en los otros cuatro países latinoamericanos 
(Argentina, Brasil, México y Chile) con mayor producción científica. Evidenciándose que en todos ellos las políticas de incremento de la productividad han sido más eficientes que en Venezuela.

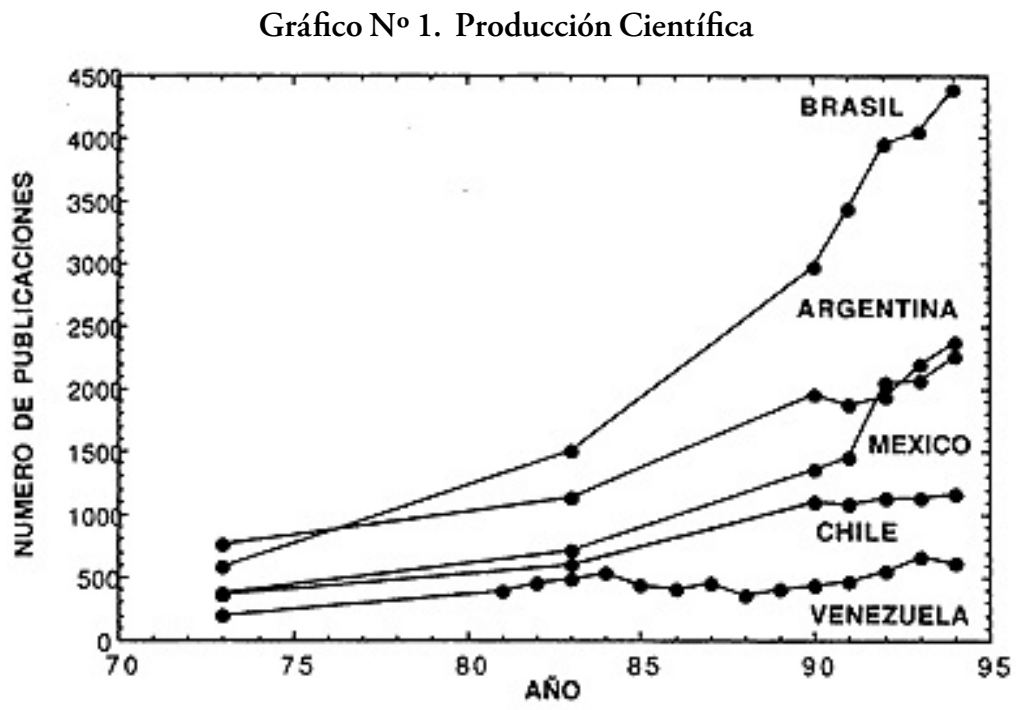

Fuente: (Bemporad, 1991; Gaillard, 1989; Rangel, 1995)

Los resultados que se señalan en los Gráficos No1 y No 2 demuestran que entre los ańos 1991 y 2003, la contribución de los países latinoamericanos en la producción científica y global también creció, los gobiernos construyen puentes efectivos entre las políticas científico-tecnológicas y las políticas de recursos humanos y educación superior. Las universidades públicas ganarán espacio relativo en la producción científica básica y aplicada por sobre los institutos independientes gubernamentales y tiende a asociarse con la formación de postgrado concentrada en las universidades de investigación y formación doctoral. Balán (2002). No obstante, Venezuela no logra superar los niveles enfrentados por la sociedad y se mantiene por debajo de países como Argentina, Brasil, México y Chile. 
Formación del investigador más allá de un currículo.

Gráfico No 2. Producción global

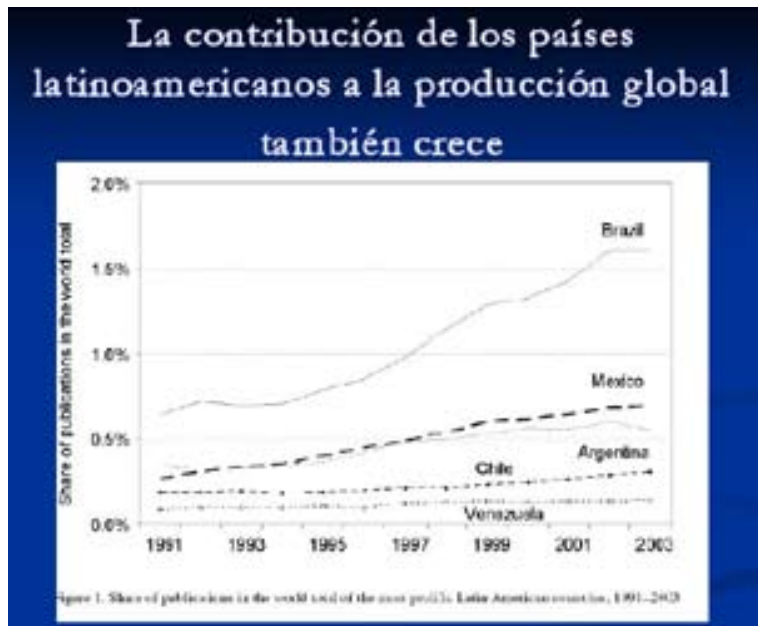

Fuente: Balán 2002

En posteriores investigaciones realizadas por Yarzábal (2013), demuestra que la investigación científica venezolana, atraviesa por uno de sus momentos más críticos. Para comprobarlo, basta con observar las tendencias más recientes de los indicadores utilizados para evaluar tal actividad. En este caso, se trata del número de artículos científicos publicados por nuestros investigadores en revistas internacionales, arbitradas e indexadas.

Gráfico No 3. Número de artículos científicos (1982- 2012)
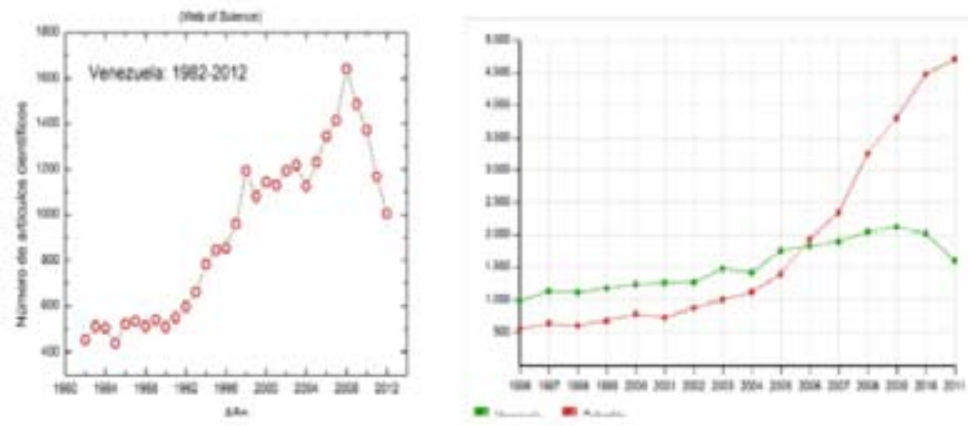

Fuente: Yarzábal (2013) 
Las evidencias expresadas en la gráfica anterior reflejan que la curva de crecimiento de los artículos científicos en Venezuela disminuyen en comparación con Colombia, a pesar de los esfuerzos que el Estado Venezolano viene implementando, entre los cuales se destacan el Programa de Estímulo a la Innovación e Investigación (PEII), que tuvo como resultado en el año 1990-2012 un total de 10.256 investigadores acreditados, en el año 2013 aumentó la participación con 21.949 investigadores en el Registro Nacional de Innovación e Investigación (RNII), arrojando un resultado de 7.800 renovaciones y 3.200 nuevos aspirantes. A pesar del incremento cuantitativo de investigadores acreditados por el PEII, este parece estar distanciado a la realidad en cuanto a la producción científica, lo que hace formular la siguiente interrogante ¿De qué manera el Estado Venezolano coadyuva a la formación de investigadores desde el pregrado? Los resultados de las investigaciones y la formación de los investigadores en las universidades son insuficientes para alcanzar lo expresado en el Plan de la Patria , Objetivo 1.5, sobre el desarrollo de las capacidades científicas y tecnológicas vinculadas a las necesidades del pueblo, donde se desprende que será a través de la educación que lleve el conocimiento de estos aspectos para convertir a Venezuela en un país potencia.

Así mismo, en datos más recientes, al comparar la inversión en materia de Ciencia y Tecnología y la posición que tiene la Universidad Venezolana mediante el "Webometrics Ranking de las Universidades del Mundo", (instrumento para medir la dinámica de las universidades en temas de publicación en revistas científicas, participación en redes científicas internacionales, presentación y contribución científica online, entre otros elementos). Venezuela (Universidad de Los Andes) ocupaba para enero 2014, la posición 46 de Latinoamérica y 1011 del ranking mundial. En la actualidad, agosto 2016, se posiciona en el lugar 62 de Latinoamérica y 1308 del ranking mundial. Entre las primeras diez de América Latina, siete (7) son de Brasil y la primera es la Universidad de San Pablo. (Ver la siguiente tabla). 
Formación del investigador más allá de un currículo.

Tabla N ${ }^{\circ}$ 1: Ranking de las Universidades del Mundo

\begin{tabular}{|c|l|l|}
\hline No & PAIS & UNIVERSIDAD \\
\hline 1 & Brasil & Universidad de São Paulo USP \\
\hline 2 & México & Universidad Nacional Autónoma de México \\
\hline 3 & Brasil & Universidad Estadual de Campinas UNICAMP \\
\hline 4 & Brasil & Universidad Federal do Rio de Janeiro \\
\hline 5 & Chile & Universidad de Chile \\
\hline 6 & Brasil & Universidad Federal do Rio Grande do Sul UFRGS \\
\hline 7 & Brasil & Universidad Federal de Minas Gerais UFMG \\
\hline 8 & Argentina & Universidad de Buenos Aires \\
\hline 9 & Brasil & Universidad Estadual Paulista Júlio de MesquitaFilho \\
\hline 10 & Brasil & Universidad Federal de Santa Catarina UFSC \\
\hline
\end{tabular}

Fuente: Webometrics Ranking de las Universidades del Mundo. Disponible: http://webometrics.info/es/Latin_America_es.

Las Universidades incrementan la matrícula estudiantil exponencialmente, descuidando el factor investigación, en este, solo se pretende que la producción de egresados o profesionales tiene como fin alcanzar la titularidad. Al respecto, Monasterio y Borjas (2012) señalan que la racionalización de las prácticas investigativas en la universidad venezolana objeto de su estudio, responden al cumplimiento de las reglas y logro de la titularidad, en detrimento del habitus de investigación. Entre las conclusiones a las cuales llegaron las mencionadas investigadoras es que la producción del conocimiento, a partir de la dinámica interrelacionar que subyace en los ambientes de aprendizaje en universidades ubicadas en área metropolitana de Caracas, deben apuntalar en la búsqueda una estrategia reorganizativa para la creación de espacios de conocimientos y transformación de los procesos vinculados a la investigación, privilegiando lo intelectivo ante la enunciación de normas y principios establecidos como requerimiento académico-administrativo por las instituciones y por docentes en calidad de asesor o tutor.

Al respecto, Rama (2011), afirma que la universidad venezolana, así como otras de América Latina, actúan como: "una máquina de formación de profesionales y con deficiencias en la 
formación de personal de investigación” (p.56). Este aspecto ocasiona un problema para el desarrollo de un país, siendo los futuros investigadores quienes contribuyen al progreso de la ciencia y la tecnología. En este orden de ideas, la tesis que se sostiene en este estudio es que la formación de los investigadores en las universidades venezolanas no responde a las políticas dadas por el Estado a través de los textos institucionales, por el contrario, está asociada a una diversidad de elementos internos y externos que subyacen en la realidad institucional. Se trata, en definitiva, de una formación dinámica, producto de los intercambios y experiencias del estudiante con el entorno sociocultural de cada universidad. De allí, surge la interrogante de conocer ¿Qué elementos gnoseológicos permiten interpretar la formación de los investigadores desde el contexto normativo del currículo y los escenarios sociales de los docentes y participantes que intervienen en los programas de las Universidades Experimentales?

En esta línea, el objetivo general de este estudio fue interpretar la formación de los investigadores desde el contexto normativo del currículo y los escenarios sociales de los docentes y participantes que intervienen en los programas de las universidades experimentales en Venezuela. Entre las limitaciones encontradas en el desarrollo de la investigación, se encontraron: la poca accesibilidad de los documentos curriculares de las universidades que fueron objeto de estudio para su interpretación. Sin embargo, la metódica del estudio permitió seleccionar unas muestras que no son representativas, sino significativas, la importancia de la significatividad, por encima de la representatividad es una condición de la muestra. Además, está centrada en contextos reducidos, el énfasis es en el valor de las palabras, más que los datos estadísticos. En cuanto a los antecedentes más destacados son los realizados por Fernández (2016), en su trabajo titulado la Deconstrucción del currículo en la formación del investigador; cuyo propósito fue generar un corpus de enunciados teóricos que contribuyó a interpretar el proceso formativo del investigador en las universidades en Venezuela, partiendo que la razón de ser de estas universidades es la producción de conocimiento. En esta dirección, se sustentó en una investigación teórica, genealógica y crítica, orientada a deconstruir el currículo como texto institu- 
cional que norma el proceso de formación del investigador en los niveles meso y micro de las carreras de pregrado de en las universidades públicas del área Metropolitana.

Este estudio desde la perspectiva Gadameriana, permitió recuperar la connotación hermenéutica del lenguaje y la tradición como racionalidad dominante en el proceso formativo, se cristalizó así, la lógica de la comunicación y el sistema simbólico que dieron cuenta de la primacía de la experiencia de los docentes investigadores en el proceso formativo del estudiante, ante la autoridad del currículo. Finalmente se generó un corpus de enunciados teóricos del proceso formativo del investigador en las universidades públicas con una mirada integral como elementos para generar una innovación educativa. Otra investigación resaltante fue la realizada por Briceño \& Meléndez (2012), titulada "La investigación como proceso de construcción social del conocimiento", se plantearon como propósito develar los procesos de construcción social del conocimiento de los docentes del Instituto de Tecnología del Oeste "Mariscal Sucre" (IUTOMS). Se pudo evidenciar un desmontaje del imaginario investigativo que soporta el proceso de construcción del conocimiento, al mismo tiempo que dibujan opciones de cara a las nuevas necesidades y nuevas utopías con el compromiso de todos los actores en la configuración de la dinámica en la investigación universitaria.

De esta manera, se develaron los procesos de construcción social del conocimiento de los docentes investigadores, visto este desde su contexto, su interacción, su relación dialógica e intersubjetiva y su encuentro con otros investigadores. Para comprender e interpretar los procesos de investigación de los docentes fueron directamente a la realidad, a la práctica cotidiana, interacciones, angustias, creencias y a partir de allí, generaron elementos teóricos que sustentan la construcción social del conocimiento. Estos elementos fueron: a) praxis del docente investigador, b) docente investigador, c) fuentes de información como ecosistema institucional y d) procesos institucionales de investigación. Concluye el estudio enfatizando que la investigación es un proceso de construcción social del conocimiento, dado que la investigación científica es un acto social que se da a partir de la acción colectiva, reconociendo el carácter histórico de formación de conocimientos porque ellos apuntan hacia la escuela 
de pensamiento dentro de la cual se ha formado el investigador. Otra experiencia vinculada con la investigación lo presenta Terán (2012) que tuvo como propósito perfilar la arquitectura de un diseño curricular desde una perspectiva compleja y transdisciplinar para lo cual, se evaluaron los diferentes métodos calificados para el diseño curricular, con la intención de determinar su aplicabilidad en la construcción de una nueva estructura que tendría el plan de estudios. Así como también, se recrearon los diferentes matices sensoriales a objeto de precisar la diferenciación óptica ante una misma situación de estudio, luego se analizó el espacio de convergencia entre las distintas disciplinas al reelaborar sus propios constructos, a fin de promover el progreso científico y técnico. En síntesis, la investigadora concluyó que el diseño curricular se ha de concebir como la construcción social en la que el sujeto del currículo es un ser social. Histórico, concreto y reflexivo, poseyendo un sistema de creencias, que muestra una relación dialógica entre el docente y el discente, reconociendo que los estudiantes interactúan con sus homólogos, con los profesores y con su propio pensamiento.

\section{La formación del investigador}

En la actualidad, la reflexión pedagógica necesita ser flexible, abierta, transdisciplinaria y multimetodológica, especialmente permeable a lo humano, su misión esencial es la de generar humanidad, facilitar que las personas se fomenten a la altura de su cultura y de su época y le den sentido a su vida. Para Flórez (1999), la educación se refiere a la interacción cultural, al proceso social mediante el cual una sociedad asimila a sus nuevos miembros incorporándolos a sus valores, reglas, pautas de comportamiento, saberes, prácticas, ritos y costumbres que la caracterizan. Esta definición marca el inicio del estudio, por cuanto la formación como el principio de humanización de los individuos que se concreta a medida que se imbrican en la educación y la enseñanza. La formación es la cualificación y el avance que logran las personas, sobre todo en sensibilidad, inteligencia, autonomía y solidaridad. En este sentido, la enseñanza es una actividad educativa más específica, intencional y planeada para facilitar que determinados individuos se apropien y elaboren 
con creatividad cierta porción del saber o alternativas de solución a algún problema en aras a su formación personal.

Así pues, compartimos la postura de Flórez (1999), al interpretar que la formación es eje y principio organizador de la pedagogía como disciplina en construcción y también es el propósito y resultado esencial de la enseñanza. Se refiere al proceso de humanización que caracteriza el desarrollo individual a medida que el ser humano se apropia de la experiencia de la sociedad a través de la cultura y de la ciencia y participa en las prácticas de sobrevivencia y convivencia de la comunidad de la que hace parte. De lo expuesto, no se escapa la pedagogía como disciplina en construcción la cual está referida a un conjunto de enunciados que configuran alrededor del concepto de formación como principio de teorías, conceptos, métodos, modelos, estrategias y cursos de acción pedagógica que pretenden entender y cualificar la enseńanza y el aprendizaje. La tarea pedagógica tiene que convertirse en una elaboración hermenéutica que traduzca e interprete públicamente el mundo de las ciencias en su proceso y en sus resultados al mundo propio de los aprendices.

En este sentido, Flórez (1999), define al profesor investigador como aquel que enseńa desde la duda, desde la pregunta, desde lo que no sabe a ciencia cierta, desde la incertidumbre, desde la ignorancia propia de los alumnos. La finalidad de su enseñanza no es el dominio y la seguridad, sino la búsqueda incansable, la intención de armar mejor el pensamiento para avanzar por el camino del espíritu inteligente, no se cansa de explorar el mundo y no se estanca, ni paraliza. Igualmente, el profesor investigador es el que asume una actitud hermenéutica que distingue al indagador, en cuanto no mira el mundo desde un lugar privilegiado y absoluto, como si no tuviera condicionamientos como los demás seres humanos, como si no tuviera ataduras sociales y culturales. El investigador cuando enseńa sabe que su primera tarea es interpretar el fondo histórico, social y cultural que lo acompańa, desde su experiencia sociocultural los alumnos deben descifrar en el aula los signos de los tiempos, de la época histórica, de la cultura y de su vida cotidiana e identificarlos de manera reflexiva los rasgos y características estructurales de la existencia empírica propia y de los alumnos.

Por consiguiente, el profesor investigador asume la actitud dialéctica del indagador que enseńa a sus alumnos mediante la búsque- 
da y el cambio metódico de posición o perspectiva que le permite alcanzar sentido permanente de superación de todo punto de vista parcial, de toda perspectiva unilateral. Cuando el profesor indagador enseńa un conocimiento verdadero coordina coherentemente más puntos de vista, más perspectivas y aspectos del fenómeno estudiado y está abierto a la crítica de una comunidad intersubjetiva de observaciones posibles. Finalmente, el profesor investigador es el indagador que enseña, no evalúa para la oficina de registro de calificaciones, sino para encontrar explicaciones cuando los estudiantes dudan de sus creencias y se atreven a pensar, a cambiar sus posiciones sobre las cosas, a discutirlas y a coordinar varias perspectivas hasta sintetizar una nueva, más completa y compleja, que puede coincidir o no con la posición del texto o del profesor; además, es un docente a tiempo completo, registrará información invaluable en sus archivos que puede alcanzar niveles de categorización e interpretación cognitiva si dispone de un protocolo inteligente para el análisis, la investigación y el mejoramiento de su enseñanza.

\section{La investigación desde currículo}

El currículo para Fernández (2010), representa una especie de reducción e interpretación de los valores, creencias e intereses de la sociedad en un ámbito eminentemente político e ideológico para determinar las formas concretas bajo las cuales se deben instrumentar los fines de la educación que finalmente se expresan en los diseños curriculares. Sin embargo, García (2005) señala que la educación se ha centrado en una visión única que es reconocida como la forma verdadera de la investigación, una enseñanza libresca, memorística, verbalista, transmisora de datos fragmentarios e informaciones puntuales que lleva a una comprensión de la ciencia descontextualizada del mundo cotidiano y de las necesidades reales. Por consiguiente, nos enfrentamos a una educación descontextualizada en la formación de los investigadores, partiendo que el currículo universitario deviene de las políticas del Estado venezolano que rige la formación de los educandos en las universidades.

En este sentido, la tendencia a mirar principalmente los aspectos más puntuales de la formación docente sin llegar a la reflexión crítica con respecto a los modelos curriculares (los explícitos y los 
ocultos) y a los modelos didácticos (muchas veces propiciadores de la simple reproducción que se asocia con la educación bancaria) que orientan dicha formación, sin duda, limita el entendimiento de la vinculación de un determinado tipo de formación con dinámicas socioculturales más amplias. La importancia de entender la didáctica y entender el currículo como condición necesaria para una práctica pedagógica reflexiva, es decir, con conciencia del papel que los educadores y las educadoras juegan en las dinámicas sociales, ha sido elocuentemente argumentada por Álvarez (2005).

De esta manera, la investigación como proceso integrado del currículo, direcciona y entrecruzan los procedimientos del trabajo científico con la disciplina donde se forma, orientando el proceso de enseñanza aprendizaje mediante el manejo de información, conceptos y contextualización de hechos, situaciones y problemas en interacción con los procesos básicos e integrados de las ciencias formales y sociales, pero sobre todo sin descuidar el abordaje investigativo en cada una de las unidades curriculares, donde los estudiantes aprenden a reflexionar; mediante la indagación sistemática, análisis crítico, clasificación, elaboración, reconstrucción y aplicación de información, procesos estos que se cruzan en el aprendizaje e investigación.

\section{Método}

El abordaje del presente estudio concibió al ser como objeto de conocimiento. Por consiguiente, pudo apoyarse en lo planteado por Santos y Madureira (2001) cuando expresan que el "enfoque cualitativo", como un tipo de metodología se refiere, en su más amplio sentido, a "la investigación que produce datos descriptivos: las propias palabras de las personas, habladas o escritas y las conductas observables" (p. 20). Así pues, la interpretación del texto por parte del lector está sujeta no solo a la recuperación de la información semántica que el texto posee, sino también a la introducción de todos aquellos elementos de lectura que el sujeto puede poseer incluido dentro de lo que hemos llamado competencia textual, desde el supuesto cultural e ideológico, los sistemas de creencias, las estructuras pasionales, entre otros. (Lozano, 2004). En la siguiente gráfica se muestra el proceso del método empleado: 


\section{Gráfico No 4. El método}

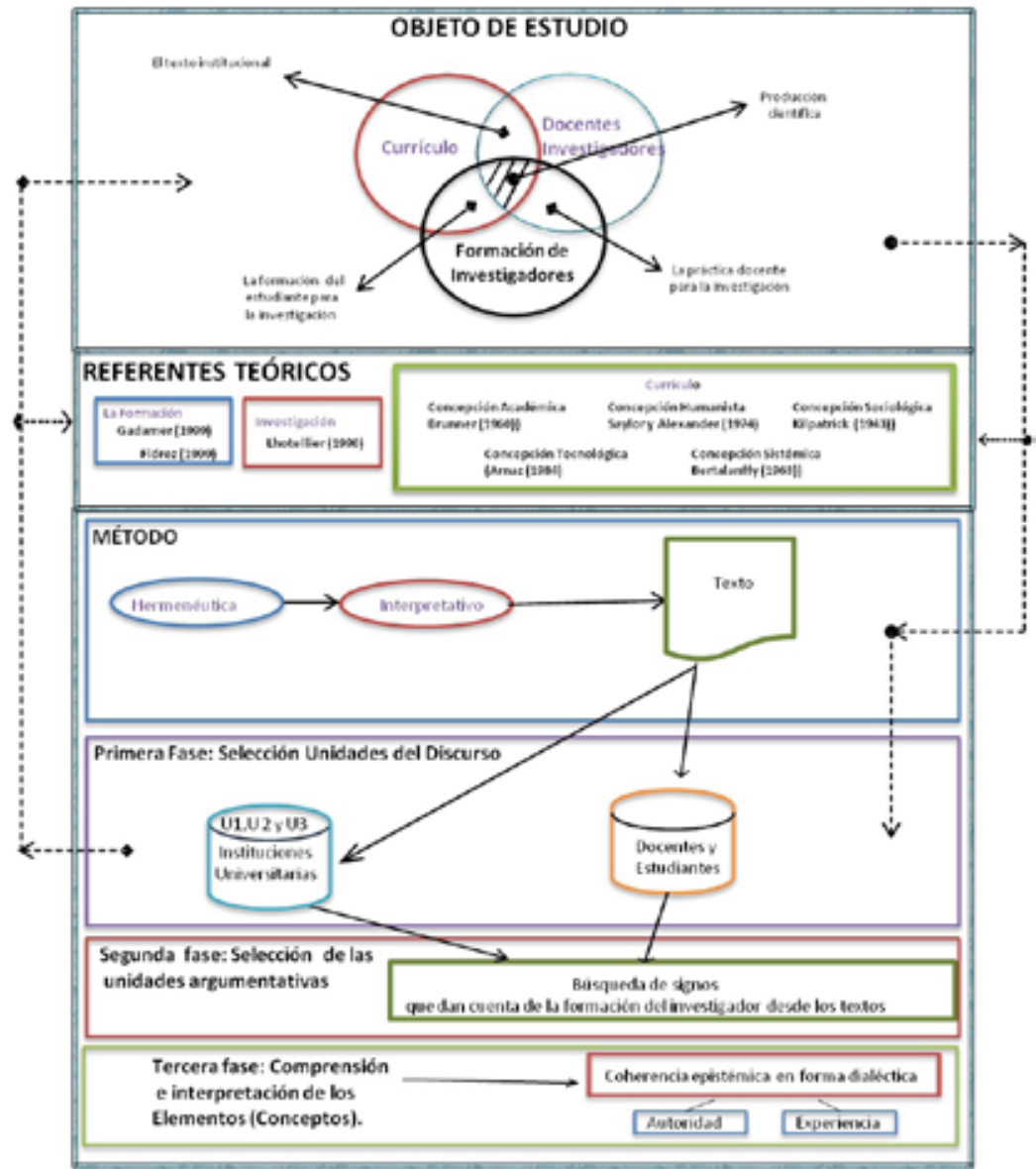

Fuente: elaboración propia

\section{Diseño}

Siguiendo a Bravo (1999), y teniendo en cuenta la naturaleza de la investigación, se asumió un diseño teórico o bibliográfico y otro de carácter empírico. Los primeros serían los que se refieren a teorías u obras sobre formación de investigadores y el currículo. En lo que respecta el calificativo de bibliográfico porque son las teorías expuestas en libros de la realidad que estudian, que se entende- 
rán normalmente de carácter no experimental. El texto concebido como el currículo viene a formar una parte fundamental en la formación del investigador, donde este currículo debe responder a los requerimientos de la época, el tipo de sociedad, región y país que se reclama a las universidades en cuanto a la formación de los recursos humanos profesionales necesarios para el desarrollo social. Por otra parte, se conciben los investigadores y estudiantes de las universidades, como parte de la realidad objeto de estudio.

\section{Participantes}

Esta investigación estuvo enmarcada en la perspectiva interpretativa, por consiguiente, recurrió a la noción de muestras significativas. La importancia de la significatividad, por encima de la representatividad, es una condición de la muestra en donde el énfasis se hace en el valor de las palabras, más que en los datos estadísticos. Patton (1990), señala que el "muestreo intencional" de tipo "intensivo" para seleccionar los informantes claves, que según son aquellas fuentes que contienen la información significativa y abundante acerca de la situación de estudio y de los eventos involucrados. También se recurre a lo planteado por Bonilla \& Rodríguez (2005), que en los estudios cualitativos la muestra no se selecciona, sino que se configura; es decir, se va estructurando a través de las diferentes etapas del proceso de recolección de datos. "Más que representatividad estadística, lo que se busca en este tipo de estudios es una representatividad cultural” (p. 134). Además, el estudio está centrado en contextos reducidos. En este orden, en un primer momento se seleccionan tres (3) universidades para el desarrollo de la investigación: La primera una (1) universidad experimental, denominada U1, otras dos (2) universidades autónomas, denominadas $\mathrm{U} 2$ y U3 (véase Tabla $\mathrm{N}^{\circ}$ 2), donde se seleccionaron los tres (3) textos académicos (currículos) de estas universidades. Considerando los siguientes criterios en la selección: carreras con formación de pregrado en el área de educación e ingeniería, aprobadas antes los entes ministeriales: Consejo Nacional de Universidades (CNU), con cohortes de egresados y con oferta académica. 
Tabla No 2 Universidades

\begin{tabular}{|l|l|l|}
\hline UNIVERSIDADES & CARACTERIZACIÓN & $\begin{array}{l}\text { TEXTOS } \\
\text { INSTITUCIONALES }\end{array}$ \\
\hline U1 & Experimental & $\begin{array}{l}\text { Licenciatura en Educación } \\
\text { Ingeniería }\end{array}$ \\
\hline U2 & Autónoma & Licenciatura en Educación \\
\hline U3 & Autónoma & Ingeniería \\
\hline
\end{tabular}

Fuente: elaboración propia

En un segundo momento, se seleccionaron cinco participantes: Docentes y estudiantes. En la selección de los entrevistados se consideraron los siguientes criterios: docentes ordinarios de las instituciones objeto de estudio, con reconocimiento institucional por participación en investigaciones, tutor de trabajos especiales de grado y/o de proyectos en comunidades, publicación de artículos en revistas arbitradas y docentes PEII. Igualmente, se seleccionaron cinco (05) sujetos reconocidos como docentes investigadores en universidades experimentales y autónomas, con potencial para develar los conceptos y elementos emergentes, así como también cuatro (04) estudiantes cursantes de los últimos semestres y recién graduados del área de Ingeniería y de Educación, provenientes de universidades experimentales y autónomas. Tal como lo establece Glaser \& Strauss (1967), las entrevistas semiestructuradas "se realiza para descubrir categorías y sus propiedades, y para sugerir las interrelaciones dentro de una teoría" (p. 62).

Estos docentes entrevistados destacados por el área investigativa provenientes de las universidades autónomas y experimentales del objeto de estudio, fueron denominados: Investigador 1 (I1), Investigador 2 (I2), Investigador 3 (I3), Investigador 4 (I4) y Investigador 5 (I5) (véase Tabla $\mathrm{N}^{\circ} 3$ ). Y los estudiantes cursantes de estas carreras: Ingeniería y Educación, fueron denominados: Estudiante 1 (E1), Estudiante 2 (E2), Estudiante 3 (E3) y Estudiante 4 (E4). Considerando que estos investigadores y estudiantes, viven y se desarrollan en un contexto histórico social determinado, actuando a través de tales experiencias investigativas en las áreas de educación e ingenierías. Asumimos la postura de Gadamer (1999), la persona a la que llamamos experimentada no es solo alguien que se ha hecho el que es a través de experiencias, sino también alguien que está abierto a nuevas experiencias. 
Formación del investigador más allá de un currículo.

Tabla Na 3. Distribución de los investigadores según tipo de institución

\begin{tabular}{|l|l|}
\hline INVESTIGADORES & INSTITUCIÓN UNIVERSITARIA \\
\hline I1 & Experimental \\
\hline I2 & Experimental \\
\hline I3 & Autónoma \\
\hline I4 & Autónoma \\
\hline I5 & Experimental \\
\hline Total & 05 \\
\hline
\end{tabular}

Fuente: elaboración propia

Tabla Na 4. Distribución de los estudiantes según tipo de institución

\begin{tabular}{|l|l|}
\hline ESTUDIANTES & INSTITUCIÓN UNIVERSITARIA \\
\hline E1 & Experimental \\
\hline E2 & Experimental \\
\hline E3 & Autónoma \\
\hline E4 & Autónoma \\
\hline Total & 04 \\
\hline
\end{tabular}

Fuente: elaboración propia

\section{Instrumentos}

Para realizar la exégesis hermenéutica de los textos académicos, se recurre a lo expuesto por Catoggio (2012), que "la hermenéutica filosófica y la praxis es el elemento irrebasable o último que otorga sentido a los conceptos que se utilizan para designar el mundo la praxis vital desde donde emergen" (p. 106). En este los significados de las palabras son un efecto de las relaciones pragmáticas que tienen los individuos en su trato cotidiano con las cosas y con otros individuos. Se trata entonces, de llegar al sentido (como elemento del proceso hermenéutico) el cual se construyó a partir de una primera proyección de los significados propios del sujeto estudiado; para después, en sucesivas interpretaciones, ir descubriendo otros elementos, siempre en relación con el entorno y traducido en términos de relaciones intersubjetivas y significados de la formación del investigador. 
Igualmente, se utilizan entrevistas semi estructuradas, las cuales define Grinnell y Unrau, citado por Sampieri, Collado \& Lucio (2010). "Se basan en una guía de asuntos o preguntas y el entrevistador tiene la libertad de introducir preguntas adicionales para precisar conceptos u obtener mayor información sobre los temas deseados" (p. 418). Desde el punto de vista del método, es una forma específica de interacción social que tiene por objeto recolectar datos para una investigación, con la finalidad de aportar datos de interés, estableciendo un diálogo peculiar, asimétrico, donde una de las partes busca recoger informaciones y la otra es la fuente de esas informaciones.

\section{Procedimientos}

En una primera fase se revisan los textos institucionales (currículos) para entender el abordaje científico en la formación del profesional, los métodos de investigación y las fuentes de información utilizadas, en algunos casos se revisaron libros, artículos y fuentes electrónicas con la intención de comprender el proceso de enseñanza y aprendizaje en la formación del investigador. Posteriormente, se revisan las entrevistas elaboradas en un formulario de Google Drive, el cual registra la información en una hoja de cálculo alojada en el Google, esta fuente de información permite comprender los ámbitos de actividad científica procedentes de la experiencia del quehacer investigativo de los entrevistados (Docentes investigadores y estudiantes).

De esta selección de unidades argumentativas surgen dos conceptos: uno proveniente de los textos institucionales, denominado autoridad, derivado de los elementos teóricos de la fundamentación ontológica, epistemológica y axiológicas, objetivos de la carrera, perfil de egreso, plan de estudio, programas y líneas de investigación. El otro concepto proveniente de los textos de los entrevistados: la experiencia, la cual surge de los elementos provenientes de tres (03) preguntas generadoras a los docentes y estudiantes que permitieron conocer la determinación del currículo para la formación de investigadores. Una vez seleccionada las unidades de argumento se procedió a la comprensión de los conceptos. Finalmente, se desarrollaron los aspectos más importantes de la investigación en 
forma recursiva con la realidad observada: las relaciones e interrelaciones que subyacen en el entramado cultural del currículo y la investigación en torno a la formación del investigador.

\section{Resultados}

A partir de los currículos, asumidos como textos institucionales desde los significados que irrumpieron el sentido de los diseños curriculares de las tres (03) universidades objeto de estudio, identificadas bajo la siguiente denominación: Universidad 1 (U1), Universidad 2 (U2) y Universidad 3 (U3). Además de los argumentos derivados de la experiencia de investigadores de reconocida trayectoria y de los estudiantes que dan sentido y significado a la noción de investigación. El objeto de estudio planteó que en el proceso formativo del investigador se permite hacer una deconstrucción en el plano interpretativo y hermenéutico a través del mismo, en donde el eje central no será descubrir la realidad social, sino interpretarla y penetrar en los conceptos que emergen de la interpretación de los textos, producto del análisis reflexivo del lenguaje. En esta línea argumentativa, se construyen en un primer momento dos conceptos como movimientos reflexivos para el análisis de los resultados.

Los elementos (conceptos) que integran el proceso formativo del investigador desde el currículo universitario, es comprendido desde el texto y resultado del proceso formativo producto de la tradición de las universidades en su papel productor de conocimiento. En este se deja abierta la posibilidad para el debate acerca de sus implicaciones en cuanto a la formación del investigador, el cual presenta un horizonte susceptible de explorar vistas las borrosidades que promete esta compleja y dinámica relación entre la autoridad y la experiencia en la formación del investigador. En este sentido, se tomaron como significados los siguientes aspectos: fundamentación de la carrera, objetivos de la carrera, perfil profesional de la carrera, plan de estudios, programas de estudio y líneas de investigación en los cuales surgieron puntos de encuentro y desencuentro, en la articulación y desarticulación de cada uno de estos elementos, tal como se muestra en la siguiente gráfica. 
Gráfico No 5 . Concepto de Autoridad

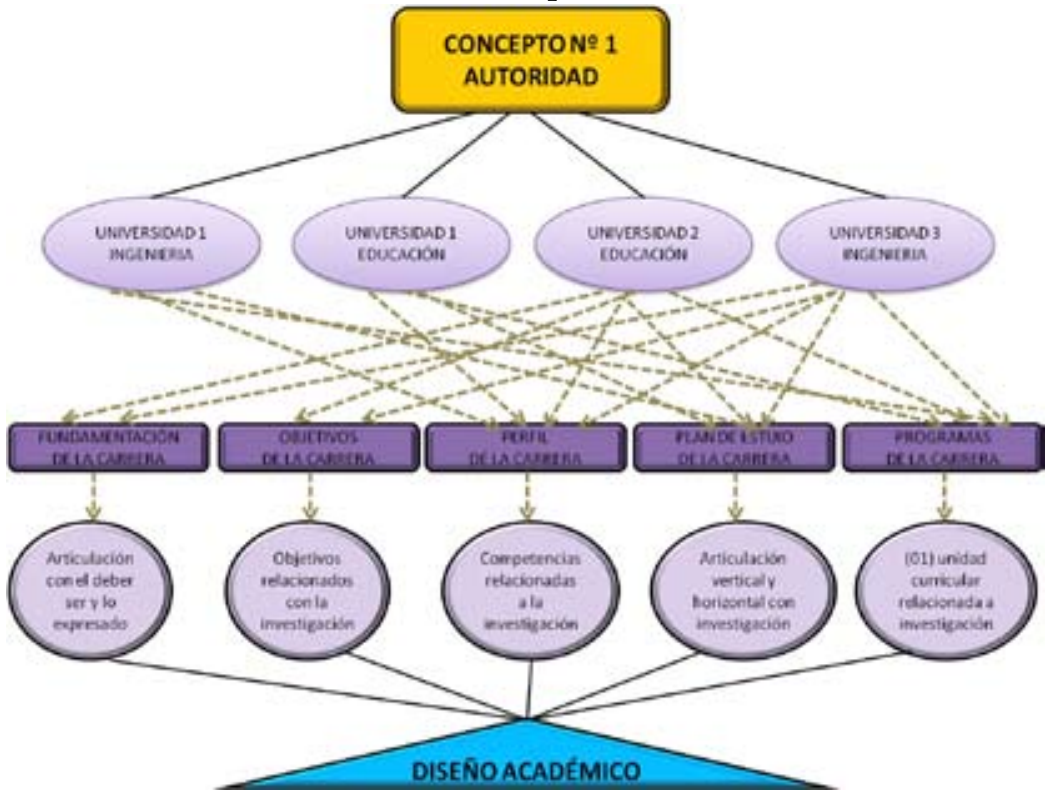

Fuente: elaboración propia

De acuerdo a las consideraciones anteriores, se interpretan los significados sobre formación del investigador que subyacen en el lenguaje de los textos en las universidades públicas del área Metropolitana, en los hallazgos encontrados se devela que la investigación no se encuentra totalmente articulada horizontal y verticalmente en el currículo, a los efectos de controlar sistemáticamente la calidad del proceso de formación, es necesario que la ciencia como actividad de producción, difusión y aplicación del conocimiento se vincule en lo curricular con el papel de la actividad investigativa como componente de la formación del educando, mediante proyectos desde sus inicios, que brinden la oportunidad de realizar prácticas vinculadas al área profesional y generar propuestas que puedan ser desarrolladas como el proyecto de investigación y finalmente la tesis de grado. 
El concepto de autoridad es una de "las instancias que operan en el fenómeno de la comprensión”, así cualquier comprensión hermenéutica del currículo, debe reconocer la autoridad que éste representa para orientar el quehacer educativo. En Gadamer, el concepto de autoridad está basado en el reconocimiento de un saber, es pues en la práctica de la vida y solo en ella donde se gana el reconocimiento de la autoridad, todo hombre afirma Gadamer (1999), "se encuentra siempre en una determinada vinculación moral y política desde la cual gana su imagen de las cosas." (p. 384). En este estudio, se asume el currículo como autoridad, porque transmite a través de la tradición las políticas y directrices que viabilizan la educación, siendo un texto escrito fundamental para la comprensión del proceso formativo del investigador. La autoridad se explica a través de seis (6) elementos que dan significado al currículo desde la perspectiva gadameriana. Por consiguiente, nos lleva a aceptar lo expresado por Gadamer (1999), que: “....nuestro ser histórico y finito está determinado por el hecho de que la autoridad de lo transmitido, y no solo lo que se acepta razonadamente, tiene poder sobre nuestra acción y nuestro comportamiento" (p. 348).

Sin embargo, la formación del investigador en las universidades venezolanas, está determinada por la autoridad que dicta el texto institucional en la fundamentación de la carrera, objetivo, perfil, plan de estudio, programas y líneas de investigación (véase gráfico $\mathrm{N}^{\circ}$ ) expresadas en los diseños curriculares de las diferentes universidades para normar el proceso de formación de los investigadores. Igualmente, la autoridad del docente, cuando ejerce el poder en el aula, poder como dispositivo de control. Dentro de este contexto, se está en presencia de unos dispositivos de control perteneciente al dominio de la legalidad impuesta desde los sistemas educativos dictados desde la figura del Estado, mediante el currículo emanado de lineamientos y directrices impuestas por el Ministerio del Poder Popular para la Educación Universitaria Ciencia y Tecnología, el Consejo Nacional de Universidades y otros diferentes dispositivos de control ejercidos sobre el cuerpo docente. No cabe duda que el poder se vuelve un dispositivo de 
control en la formación de los investigadores en Venezuela. Autoridad y poder, un binomio que atenta contra la reflexión crítica de la praxis investigativa.

Por otra parte, en el marco de la formación del investigador en el currículo no solo fue suficiente analizar los textos institucionales, sino que se recurrió también a la experiencia producto del resultado de las entrevistas comprendidas e interpretadas en los textos, que dio una mirada complementaria, su mundo vivido, el mundo del ser, su trayectoria como investigador y docente, como estudiante que experimenta durante la formación en su área profesional, en los cuales surgen los siguientes elementos: Proceso Formativo del Investigador y el Contexto. En este análisis se determinó que el currículo, su desarrollo y administración son claves importantes en el proceso de formación del investigador, siempre y cuando se asuman los procesos como complejos, que impliquen reconocer que hay más aspectos intervinientes de los que conocemos y podemos manejar, debemos reconocer que no es posible afirmar que una sola cosa determina la formación.

Tal como lo señala Pozo \& Gómez (2001) al establecer que "enseñar a investigar es enseñar a pensar, indagar, comprender, distinguir, seleccionar, argumentar razonadamente y esto se enseña más, dentro de un estilo pedagógico y una didáctica específica, que como un ejercicio de laboratorio experimental" (p. 65). Esto ratifica, que el docente con su formación, disposición y capacidad de inducir al estudiante en la construcción del aprendizaje investigativo, favorece la interconexión enseñanza-aprendizaje-investigación en contextos universitarios. Por otra parte, la motivación juega un papel importante dentro de la formación del investigador, a pesar que la motivación en la investigación es intrínseca de cada estudiante, es necesario que los docentes contribuyan a la formación inicial de los futuros investigadores, a través de un conjunto de estrategias que despierten el interés y la motivación por la investigación.

De acuerdo a los planteamientos anteriores, se configuraron una red de significados que permitieron interpretar la formación de los investigadores en el contexto normativo del currículo y los escenarios sociales de los docentes y participantes que interviene en los programas universitarios de las Universidades Experimentales en Venezuela, derivados de los hallazgos encontrados en los textos re- 
sultados de las entrevistas a docentes y estudiantes, el cual develó que no solo el currículo forma a los investigadores, sino que existen otros elementos que influyen en esta formación, tales como: docente tutor que sensibilice en el quehacer investigativo, difusión, promoción de publicaciones, convenciones y foros, ambientes de laboratorios y salas de informática para la investigación de proyectos. Así pues, Autoridad y Experiencia binomios para la formación del investigador. De acuerdo a estos elementos surge el concepto de experiencia:

\section{Elemento: experiencia}

El concepto de experiencia como elemento central para comprensión de la formación de los investigadores se construye desde los significados propios que otorgan los investigadores y estudiantes que conformaron el estudio. Siguiendo la perspectiva de Gadamer (1999), quien extrae el concepto de experiencia, afirmando que:

"La verdad de la experiencia contiene siempre la referencia a nuevas experiencias. En este sentido, la persona a la que llamamos experimentada no es solo alguien que se ha hecho el que es a través de experiencias, sino también alguien que está abierto a nuevas experiencias" (p. 431).

Lhotellier (1990), refiere que la formación es la capacidad de transformar en experiencia significativa los acontecimientos cotidianos, en el horizonte de un proyecto personal y colectivo, por lo tanto, enmarcado en esta idea, la formación de investigadores no es algo que se adquiere de una vez por todas, que es posesión de algunos o que se consigue solo con un título profesional; sino más bien, puede concebirse, como una especie de función propia del ser humano, que se cultiva y puede desarrollarse, que no está sujeta a temporalidades o edades específicas. Esta característica fácilmente puede atribuirse a la tesis de Gadamer (1999). La formación es formarse". Por tanto, el concepto de experiencia es donde comienza a percibirse el carácter ontológico de la hermenéutica. No es que la experiencia constituya el carácter ontológico de la hermenéutica, sino que es el punto del que parte esa constitución. Es aquí donde develamos en los textos de los entrevistados como se da la relación que posee el docente con respecto al estudiante y la realidad circundante del mismo, para llegar a la 
reflexión de los resultados del proceso formación para la investigación en la práctica como producto de la experiencia.

En este sentido, en el gráfico $N^{\circ} 6$ representamos gráficamente el concepto de experiencia desde sus dos (02) elementos: Proceso formativo del investigador y el contexto. Los resultados o hallazgos permitieron crear un diagrama de movimiento del proceso de formación de los investigadores en las universidades en Venezuela a través de una imagen. Esta se interpreta mediante un plano de consistencia (Ver Gráfico $N^{\circ} 7$ ), que representa los movimientos que subyacen en los niveles de concreción que el investigador creo de manera originaria, además, surge de la propia realidad y está inmerso en un horizonte histórico cultural.

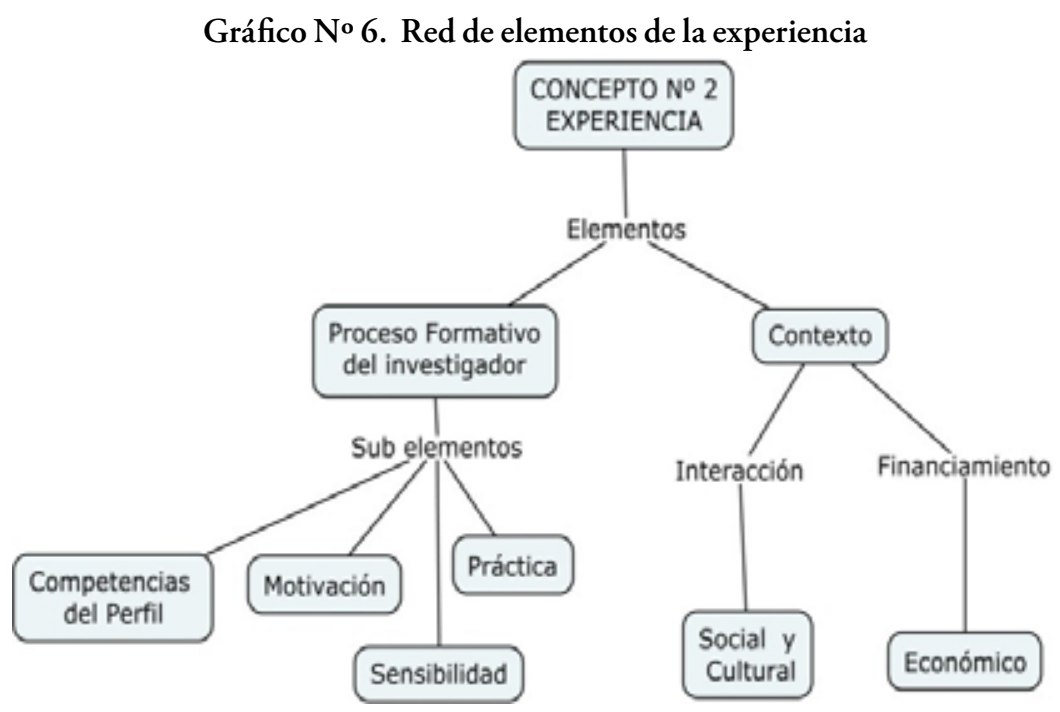

Fuente: elaboración propia 


\section{Gráfico No 7. Plano de Consistencia}

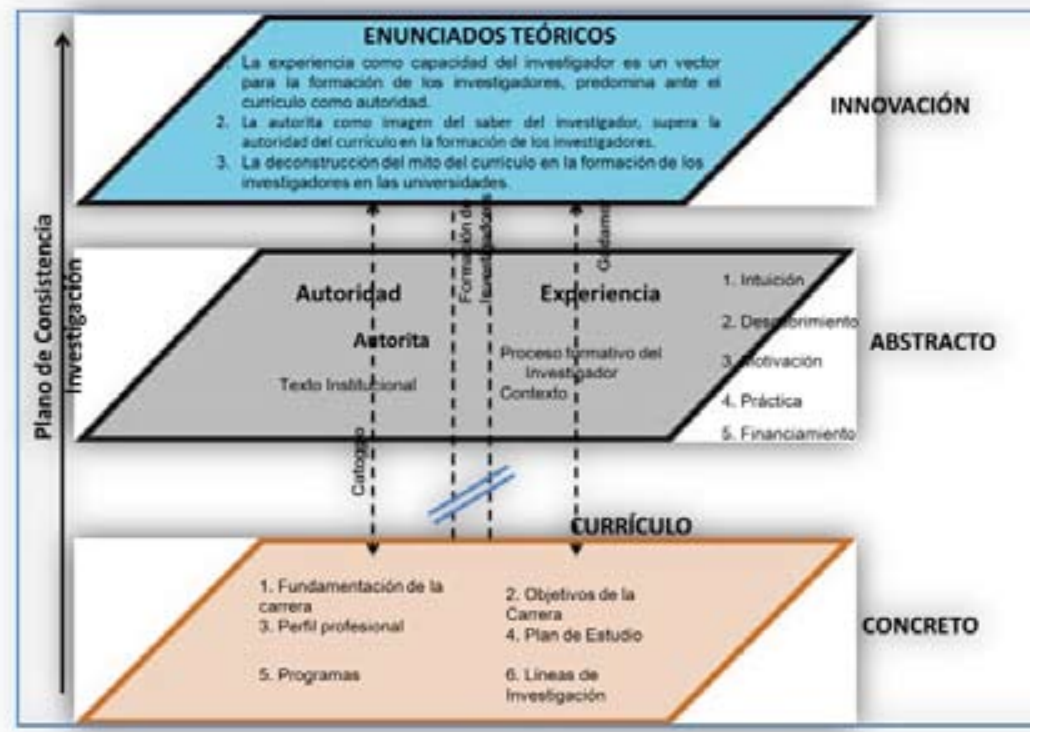

Fuente: elaboración propia

Siguiendo, los planteamientos de Deleuze (1991), expresa que no es un concepto pensado, ni pensable, sino la imagen que se da a si mismo de lo que significa pensar, hacer uso del pensamiento, orientarse en el pensamiento. Desde esta afirmación se interpreta que las diferentes modalidades de existencia de los sistemas de intensidades no atañen a idealidades transcendentes, por el contrario, son procesos de engendramiento y de transformaciones reales. Desde esta perspectiva, no se trata de cartografiar lo encontrado en los textos y en los discursos de los docentes y participantes de las diversas carreras, se asiste a la construcción de un plano que permite observar cómo es el recorrido desde lo concreto a lo abstracto y trasciende al plano de la innovación.

En este estudio, surge así el plano concreto que permite describir cada uno de los elementos que dieron cuenta acerca del texto interpretado, surgiendo así la reflexión que sirvió de punto de partida para la conexión de sus componentes con el plano abstracto, donde desmonta la idea habitual del concepto en el que el currículo es 
quien forma a los investigadores, llegando así al plano de concreción o inmanencia que proyecta una figura de conexión con ambos planos, que trasciende al plano de la innovación mediante una proyección absoluta de la inmanencia, desde un horizonte de los elementos encontrados para interpretar la formación de los investigadores en el contexto normativo del currículo y los escenarios sociales de los docentes y participantes que interviene en los programas universitarios de las Universidades Experimentales en Venezuela.

\section{Conclusiones}

En la comprensión del currículo, como proceso social que fortalece y organiza las bases educativas para el avance del conocimiento y que contempla los tres estados curriculares, tales como: diseńo, gestión y evaluación, pasa a ser un mito en la formación del investigador, debido a que existen otros elementos que determinan esa formación. Así se abre un nuevo horizonte o se crea una fusión de horizontes para repensar el proceso de formación de los investigadores en las universidades. El proceso de formación del investigador recae sobre el texto institucional (currículo) como autoridad. En su concepto, el verdadero fundamento de la autoridad es "un acto de la libertad y la razón, que concede autoridad al superior, básicamente porque tiene una visión más amplia o está más consagrado, esto es, porque sabe más." Gadamer (1999, p-384). Autoridad poco reconocida por los investigadores, en otras palabras, los estudiantes se forman con los investigadores y son estos quienes desde su experiencia coadyuvan al proceso de formación.

De lo expresado, se explica el rechazo de los investigadores al proceso de formación y desarrollo, caracterizado bajo una concepción pedagógica autoritaria que estructuralmente tiende a la formación de un sujeto pasivo, memorístico, repetidor de contenidos que son transmitidos por un docente que se formó del mismo modo, como lo afirma Gadamer que la formación se da como elemento dentro del cual se mueve quien se ha formado de este modo, la autoridad que deviene del currículo se encuentra en la crítica constante de los investigadores. La verdad y la autoridad del currículo como texto institucional, debe colocarse entre paréntesis, este texto solo responde al cumplimiento de las exigencias legales y adminis- 
trativas que demanda el Estado en materia de educación universitaria para formar los profesionales hacia el mercado laboral. Y en función de acuerdos racionales para mantener hegemónicamente, ciertos métodos y paradigmas.

Desde este horizonte, las palabras de Padrón (1992), como investigador venezolano son importante cuando alerta en el siglo pasado que la universidad e investigación, son un binomio que requiere del docente investigador para crear las comunidades científicas con una mirada del hacer científico. El investigador venezolano parte de la necesidad de que las universidades creen, desarrollen y conduzcan sistemas organizacionales, contando con esa gran potencialidad de recursos humanos que son los estudiantes y los docentes, en cuanto a posibles investigadores. Estos sistemas organizacionales deben ser capaces de analizar el mercado, de diseñar redes de problemas investigativos, de distribuir a estudiantes y docentes alrededor de las funciones y tareas planteadas según esas redes problemáticas, de asignar recursos y asistencia técnica, de promover y difundir los productos logrados y, finalmente, de realimentar y evaluar el sistema.

A manera de conclusión, las universidades tienen un papel fundamental como instituciones científicas proveedoras de conocimientos donde no solo se otorgan títulos profesionales, sino que se forman investigadores. Briceño \& Chacín (1994:91) señalan que: "La universidad tiene un compromiso con la sociedad, pues ella está colocada en una situación particularmente significativa en cuanto a la posibilidad de transformar, a través de la creación y recreación del conocimiento científico y sus derivaciones tecnológicas, la vida nacional en todos sus aspectos”. La institución universitaria es el espacio para la formación de investigadores de alto nivel y los conocimientos que en ella se generen, deben impactar necesariamente a la comunidad. Sin embargo, la realidad en las universidades venezolanas dista de las aspiraciones de todos los académicos que hacemos vida en el recinto universitario, existen muchos elementos que obstaculizan el desarrollo en la formación para la ciencia.

En general, los contenidos curriculares se organizan con una estructura, que contiene poca lógica y coherencia, lo cual limita la articulación entre pregrado, postgrado y extensión, planteándose oportunamente la ubicación de la información precisa en los con- 
tenidos asociados a los mecanismos curriculares, organizados por bloques o ejes de formación, pero quedando declarada la investigación como eje central en la formación del profesional con base en las diversas carreras que finalmente serán administradas por el docente mediante los contenidos de los programas analíticos. El desarrollo de los programas de estudio limita el pensamiento crítico y solo responden en función de la estructura lógica de las disciplinas del plan de estudios, se impone la necesidad que en estos se planteen los problemas profesionales principales y de cada uno de estos problemas principales se derivan otros, propedéuticos, que constituyen los principales proyectos a realizar por los estudiantes, así como el sistema de tareas que conduce a la solución de los mismos.

Finalmente, en el currículo deben quedar expresadas las competencias del perfil del egresado orientadas, además de lo profesional a la investigación, incorporando un componente de investigación como eje central del programa, que delineen las competencias investigativas. Pero este debe ser desarrollado a través de toda la escolaridad, no como un complemento, sino constituyéndose el foco central en la formación. Por otra parte, la experiencia en el quehacer investigativo es otro concepto que delinea la formación de los investigadores, en este se percibe el carácter axiológico y las características del contexto y del medio en el que se desenvuelve el investigador. En este proceso de formación es importante que el docente sensibilice a sus estudiantes para el trabajo investigativo, direccionando al alumno hacia los trabajos científicos, mediante la participación en los procesos educativos que conlleven a investigar, descubrir, comparar y sistematizar experiencias, guiado por el docente en su rol de orientador y brinde acompañamiento en todo el proceso educativo.

Otro aspecto determinante para la formación del investigador es el financiamiento, factor importante para la inversión en investigación, tales como: bibliotecas, suscripciones a revistas científicas, mantenimiento y modernización de equipos, laboratorios y centros de investigación, el fortalecimiento de la calidad de las revistas de investigación a los fines de lograr su acreditación, indexación e incrementación en la cantidad y la calidad de las publicaciones científicas periódicas. Así como también, el patrocinio de jornadas, exposiciones, congresos y otros eventos científicos, la promoción 
de programas y proyectos del CDCHT, la divulgación del personal docente y de investigación en el Premio Anual de Investigación PEII y la Red de investigación estudiantil.

Es importante que la investigación tiene que estar bien expresada en los diseños curriculares, vinculado a las políticas educativas de la institución universitaria, mediante los centros de investigación, Consejo de Desarrollo, Científico, Humanístico y Tecnológico de la Universidad (CCDHT) y las Coordinaciones, de acuerdo a la estructura de cada centro, a fin de concentrar experiencias de distintas especialidades con aportes que harán los centros de investigación según sus áreas fuertes, a manera de establecer transversalidad entre diferentes fuentes del conocimiento y organizar la investigación enmarcada dentro de una dinámica colaborativa; tomando en cuenta diversas áreas y sectores de interés según las necesidades locales, regionales y nacionales, permitiendo así un buen desarrollo de las condiciones que favorezcan la formación del investigador. Al cierre de esta investigación, surgen dudas sobre si: ¿¿Será necesario repensar la función de la investigación en las universidades públicas venezolanas?, ¿la investigación conduce al desarrollo o el desarrollo promueve la función de la investigación en las universidades? Y finalmente, ¿cuál es el uso que tiene la investigación que se produce en el marco de los programas académicos, aparte de ser un requisito para la obtención de títulos profesionales?

\section{Referencias bibliográficas}

Álvarez, M. \& García, L. (2005). La formación del profesorado universitario para la innovación en el marco de la integración del espacio europeo de educación superior. Disponible: www.revistaeducacion. mec.es/re337.htm [Consulta: septiembre 18, 2016].

Balan, J. (2002). Universidad e investigación científica en países en desarrollo. Buenos Aires Argentina. Disponible: http://www.mineducacion.gov.co/cvn/1665/articles-192182_archivo_ppt8.pdf Consulta: enero 18, 2014].

Bonilla, E. \& Rodríguez, P. (2005). Más allá del dilema de los métodos. La investigación en ciencias sociales. Bogotá: Grupo Editorial Norma

Bravo (1999). Tesis doctorales y trabajos de investigación Científica. 
España. 5ta edición. Editorial Panapo.

Briceño, M. \& Chacín, M. (1994). Análisis de la relación docencia-investigación en las universidades venezolanas. V Jornadas de Investigación. Universidad Nacional Experimental "Simón Rodríguez". Caracas.

Briceño \& Meléndez (2012). La investigación como proceso de construcción social del conocimiento. Caracas. Fondo Editorial Ipasme.

Catoggio (2012). La regla hermenéutica como sistema de significación y producción de texto. Argentina. Disponible: http://www.scielo.org. co/pdf/ef/n45/n45a06.pdf [Consulta: febrero 03, 2016].

Deleuze \& Guattari (1991). Qu'est-ce que la Philosophie? Paris: Minuit. Fernández, M. (2016). La deconstrucción del currículo en la formación del investigador. Tesis de Doctorado no publicada, Universidad Nacional Experimental de la Fuerza Armada Nacional, Caracas, Venezuela.

Fernández, A. (2010). Universidad y currículo en venezuela: hacia el tercer milenio. 2da edición. Vicerrectorado Académico. Comisión de Estudios de Postgrado- Facultad de Humanidades y Educación de la Universidad Central de Venezuela

Flórez, R. (1999). Hacia una pedagogía del conocimiento. Bogotá, Colombia: McGraw-Hill

Gadamer (1999). Verdad y Método I y II. Salamanca. España. Ediciones Sígueme.

García, P. (2005). El sentido de la educación como referente básico de la didáctica. Disponible: https://dialnet.unirioja.es/servlet/ articulo?codigo=1166087 [Consulta: enero 18, 2014].

Glaser, B. \& Strauss, A. (1967). The Discovery of Grounded Theory: Strategies for Qualitative Research. Chicago: Aldine.

González, Hernández \& Sanz (2003). Curriculum y formación profesional. Centro de estudios para el perfeccionamiento de la Educación Superior. Cuba.

Imbernón, F. (Ed.) (2003). La investigación educativa como herramienta de formación del profesorado: Reflexión y experiencias de investigación educativa. Barcelona: Editorial Graó.

Lhotellier (1990). La formación en investigación en el colegio de pedagogía. Un análisis metodológico en la perspectiva del plan de estudios. Cuadernos del CESU, núm. 9, UNAM, México.

Lozano, J., Peña, C., \& Abril, G. (2004). Análisis del discurso: hacia una 
semiótica de la interacción textual. Madrid: Cátedra.

Mayz \& Pérez (2002). ¿Para qué hacer investigación científica en las universidades venezolanas? Disponible: http://www.scielo.org.ve/scielo. php?pid=S1316-0872002000100007\&script=sci_arttext [Consulta: agosto 23, 2014].

Monasterio \& Borjas (2012). La producción del conocimiento desde las representaciones sociales de los Estudiantes de Postgrado. Evento científico LXII. Convención Anual de AsoVAC. Caracas. Venezuela. Del 18 al 23 de noviembre de 2012.

Padrón, J. (1992). Paradigmas de investigación en ciencias sociales. Un enfoque curricular. Papel De Trabajo, Postgrado, UNESR. Caracas, mayo.

Patton, Michael Quinn (1990). Qualitative evaluation and research methods. Newbory Park: Sage Publications.

Pozo, V. \& Gómez, J. (2001). Aprender y enseñar ciencia. Madrid. Ediciones.

Rama, C. (11-03-2011). Entrevista con Johandry Hernández. Universidad del Zulia. Disponible: en http://www.agenciadenoticias.luz.edu. ve/index.php?option=com_content $\&$ task=view\&id=2102\&Itemid= 159. [Consulta: febrero 07, 2012].

Sampieri, Collado \& Lucio (2010). Metodología de la investigación. 5ta Edición. Editorial Mc Graw Hill. México.

Santos \& Madureira (2001). Metodología das ciencias socias. Porto: Editorial Edicoes Afrontamento.

Terán (2012). Arquitectura del diseño curricular: una perspectiva compleja, transdisciplinar y postmoderna. Tesis de Maestría en Educación Mención Currículo. Universidad Pedagógica Experimental Libertador. Dirección de Investigación y Postgrado. Caracas.

Yarzábal, A. (2013). L'imagination au pouvoir o Ciencia imaginaria para universidades en tiempos de crisis. Laboratorio de Microbiología. Moleculary Biotecnología. Facultad. 